\title{
Identification of control and management strategies for LV unbalanced microgrids with plugged-in electric vehicles
}

\author{
J.A. Peças Lopes ${ }^{\text {a }}$, Silvan A. Polenz ${ }^{\text {b }}$, C.L. Moreira ${ }^{a}, *$, Rachid Cherkaoui ${ }^{\mathrm{b}}$ \\ a INESC Porto, Faculdade de Engenharia da Universidade do Porto, Campus da FEUP, Rua Dr. Roberto Frias 378, 4200-465 Porto, Portugal \\ ${ }^{\mathrm{b}}$ EPFL-Ecole Polytechnique Fédérale de Lausanne, Laboratoire des Réseaux Électriques, Lausanne, Switzerland
}

\section{A R T I C L E I N F O}

\section{Article history:}

Received 12 October 2009

Accepted 30 December 2009

Available online 22 January 2010

\section{Keywords:}

Distributed storage

Electric vehicles

Frequency control

Microgrid

Vehicle-to-grid

Voltage control

\begin{abstract}
A B S T R A C T
This paper addresses issues concerning the integration of single-phase charging devices for electric vehicles (EV) in low-voltage microgrids. Fast release energy storage is a key issue for microgrid islanding operation. EV batteries provide an additional storage capacity, which can now be exploited in order to improve MG islanding. Aiming to do so, different control strategies were developed and tested: (1) a local control approach where no communication link is required and (2) a centralized charging control solution. The local control approach is based on the measuring of EV terminal voltage and frequency in order to define the charging or discharging rates of the batteries. The centralized control strategy allows balancing single-phase loads connected to the microgrid by adapting the charging rates of the EV storage devices. Simulation results show that EV batteries can actively contribute for voltage balancing and frequency control during islanding operating conditions.
\end{abstract}

() 2010 Elsevier B.V. All rights reserved.

\section{Introduction}

The electric power systems industry is about to face a major new challenge: future massive integration in the electric grid of plug-in electric vehicles (EV). The stimulus for this change is that electricity is likely to become the preferred energy vector for a new generation of road vehicles. This new trend will be established in a scenario characterized by large penetration of renewable power sources (RES), some of them with an intermittent nature like wind generation. The integration of these RES will involve also microgeneration solutions, being these small generation units connected to the LV grid.

There are two ways of accommodating the connection of EV in distribution grids. The first is to plan for new networks in such way that they can fully handle the new loads, regardless of the control scheme, requiring heavy investments in network reinforcements to do so. The second is to create a smart management system that fully integrates EV in the power system, exploiting also the potential of EV as energy storage devices and creating a large distributed storage infrastructure that can be used to help the system in several situations. The latter is, of course, the way that needs to be pursued.

EV will be charged with electricity from the grid and they can also provide power to the grid when parked, as discussed in the papers from Kempton and Tomić [1] and Brooks [2]. Assuming large

\footnotetext{
* Corresponding author. Tel.: +35122 209 4210; fax: +35122 2094150.

E-mail address: cmoreira@inescporto.pt (C.L. Moreira).
}

penetration of EV in the transportation sector, a considerable volume of storage capacity will then be connected to the grid during most of the time [1], such that it can be used to help managing the electric power system, delivering namely ancillary compensation services, leading to the vehicle-to-grid (V2G) concept. Three elements are required in order to develop further this concept: a smart interface with the grid, communication with the grid operator to receive control signals and metering solutions to account for EV energy exchanges with the grid.

Progressive replacement of conventional vehicles by EV will require two types of interfacing structures: (a) charging stations used to charge fleets of EV or to charge EV that require fast charging or (b) domestic or public individual charging/grid interface points for slow charging. In this paper only EV connected to LV grids are addressed, considering the adoption of solution (b) in LV grids.

Implementing a smart EV management involves dealing with the concepts of SmartGrid and Microgrid (MG) [3], namely if EV are to be connected to LV networks. EV will interact with the electrical systems of the future, where distributed intelligence will be present, and are likely to reduce the need for conventional large energy storage devices that are required to deal with large scale intermittent renewable generation.

Storage capability has been identified as a key issue to allow for successful MG islanding operation. Different technologies, such as batteries, flywheels, or supercapacitors, can be used for this implementation. In the previous developments of the MG concept, storage units were considered to be interfaced with the grid through Voltage Source Inverters, responding proportionally to 


\begin{tabular}{|ll|}
\hline \multicolumn{2}{|l|}{ Nomenclature } \\
EV & Electric Vehicle \\
LC & Load Controller \\
LV & Low Voltage \\
MG & MicroGrid \\
MGCC & MicroGrid Central Controller \\
MC & Microsource Controller \\
MMO & Multi-Master Operation \\
RES & Renewable Power Sources \\
SMO & Single Master Operation \\
V2G & Vehicle-to-Grid \\
VC & Vehicle Interface Controller \\
VSI & Voltage Source Inverter \\
VUF & Voltage Unbalance Factor \\
\hline
\end{tabular}

frequency changes using a droop control mode approach as described in Refs. [3-5]. In previous research these units were supposed to be installed close to the MV node (as described in Fig. 1), since they may release considerable amounts of power during transient phenomena.

In this paper an innovative approach is described by integrating into MG the storage capability of EV, involving the adoption of a specific control approach that contributes to balance load and generation in islanding conditions and to compensate voltage unbalances in normal and islanding modes of operation. In this way the presence of a large number of distributed storage devices that belong to EV can be exploited to consolidate the MG and the multi-microgrid concepts [4] and bring additional resilience to the distribution grid by allowing islanding procedures to be successfully implemented.

\section{MG architecture and EV}

A MG is a low-voltage network with several microgeneration units, controllable loads, storage devices and a hierarchical control system [4], such that the MG can be operated either in a normal interconnected mode or in a stand-alone mode [5,6]. A physical architecture of a MG with EV connected to it is shown in Fig. 1.

The MG is controlled by a MicroGrid Central Controller (MGCC) installed at the MV/LV substation, including some key functions and heading the local grid control scheme. A communication infrastructure between the MGCC and the local controllers (LC and MC for load controllers and microsource controllers, respectively) needs to be established. Regarding the MG concept defined in Ref. [5], a new set of active storage devices has now been included - electric batteries belonging to EV, as described in Fig. 1. These batteries are supposed to be single-phase grid connected, through a smart power electronic interface with a specific control approach - the vehicle interface controller (VC). The implemented control concept allows a smooth transition between the two possible MG operation modes (interconnected and stand-alone mode), as well as participation in frequency and voltage balancing during islanding operation mode. The MGCC may interact also with a higher hierarchical control level taking care of a MV grid with several other MGs as described in Ref. [4].

The EV grid interfaces can be located on-board the vehicle or outside in any node of the grid. It was also assumed that when in charging mode the desired operating point is the rated charging power of the battery. Other operating situations as described in Ref. [7] can also be imagined.

\section{Voltage (un)balance}

As battery charging devices of EV are single-phase connected units that will coexist with normal single-phase loads, voltage (un)balance needs to be addressed in a MG in this scenario. The evaluation of the unbalancing is performed through the Voltage Unbalance Factor (VUF), as used in the European power quality standard EN50160 "Voltage characteristics of electricity supplied by public distribution systems", being defined as:

$\% V U F=\frac{V_{2}}{V_{1}} \times 100 \%$

where $V_{1}$ and $V_{2}$ are, respectively, the positive and negative sequence voltage values.

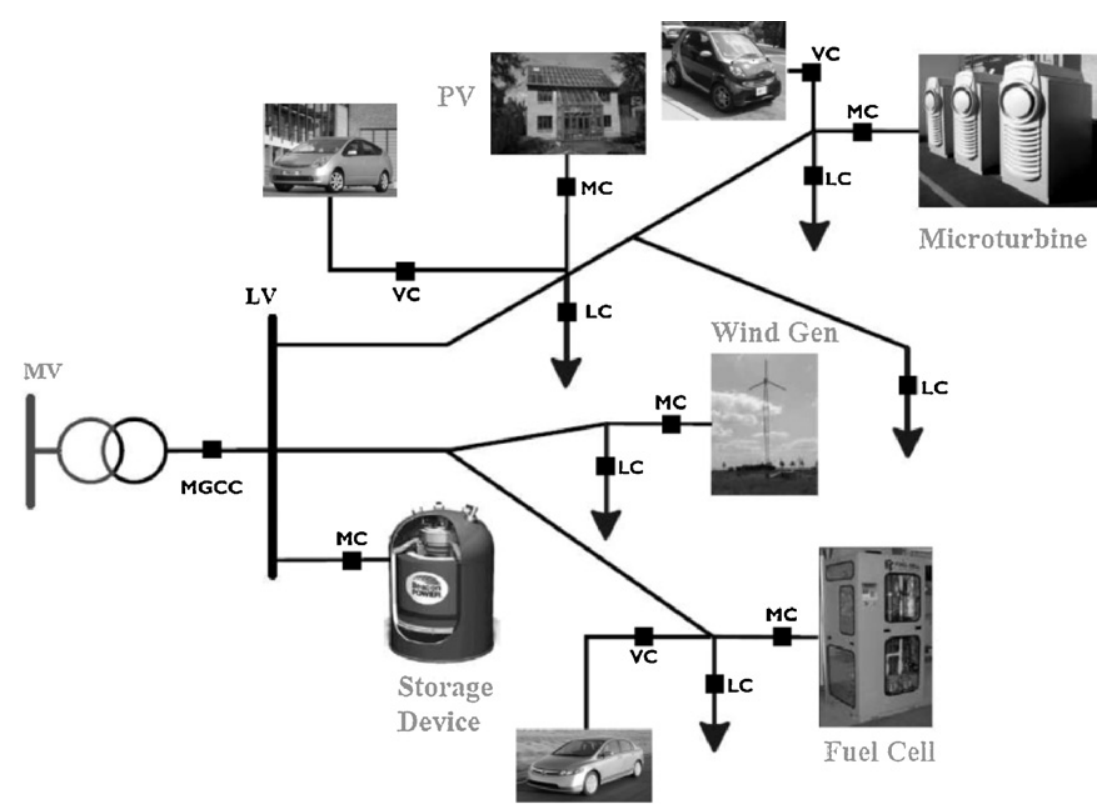

Fig. 1. MG architecture, including microgeneration, loads, electric vehicles, and control devices. 


\subsection{Correction of voltage unbalance}

Different strategies can be adopted to reduce voltage unbalance. In a classic distribution grid, static VAR compensators, or passive power filters may be used. Furthermore changing the system configuration through manual or automatic feeder switching can improve voltage balancing conditions [8]. Lasseter and Piagi suggested injecting sequence currents from inverters to rebalance the system [9].

\subsection{Measurements in unbalanced systems}

Accurate power measurements are essential for robust operation of MGs. The following approach was adopted in this research to evaluate active power for an unbalanced four-wire three-phase system. For a three-phase power system, instantaneous voltages and instantaneous currents can be expressed as instantaneous space vectors, described by (2):

$\bar{v}=\left[\begin{array}{c}v_{\mathrm{a}} \\ v_{\mathrm{b}} \\ v_{\mathrm{c}}\end{array}\right], \quad \bar{i}=\left[\begin{array}{c}i_{\mathrm{a}} \\ i_{\mathrm{b}} \\ i_{\mathrm{c}}\end{array}\right]$

The instantaneous active power of a three-phase circuit, $p$, can then be given by (3) and the reactive (non-active) power, $q$, is calculated in (4).

$p=\bar{v}^{T} \cdot \bar{i}$

$q=\left\|q_{\text {space }}\right\|=\|\bar{v} \times \bar{i}\|$

For a single-phase connection, the measurement was made based on the proposal of Burger and Engler [10]. With this approach no zero-crossing detection is required. The power is computed by using the complex apparent power split up in its real and imaginary part, as described next:

$\underline{s}=p+j q=\frac{1}{2} \underline{v} \cdot \underline{i}^{*}=\frac{1}{2}\left(v_{2}+j v_{\mathrm{i}}\right)\left(i_{\mathrm{r}}-j i_{\mathrm{i}}\right)$

where $v_{\mathrm{r}, \mathrm{i}}$ and $i_{\mathrm{r}, \mathrm{i}}$ are fictitious orthogonal components of the voltage and current, similar to the space vectors in three-phase systems.

\section{Modelling of the components}

The main objective of this work regards the evaluation of the dynamic behaviour of the MG with a large amount of controllable storage devices in charging/discharging modes belonging to EV (acting as V2G devices). Since fast transients were not a matter of concern in this research, only the main control functions of the inverters were used as described in Refs. [3-5]. Grid interface inverters belonging to microsources and $\mathrm{EV}$ batteries were then modelled by a simple control logic approach.

It is possible to distinguish two different types of inverter control: PQ control and Voltage Source Inverter (VSI) control as described in Ref. [5]. The PQ-controlled inverter is used to supply a given amount of active and reactive power to the grid. This power set point can be defined by the primary source (e.g. in solar panels) or defined externally to the microsource by a control system like the MGCC. A VSI controlled source is able to react to system demands by using information available at its terminals (frequency and/or voltage). It is capable of emulating the behaviour of a synchronous machine and providing primary frequency and voltage regulation to an islanded MG. Further details can be found in Refs. [3,5].

\subsection{V2G grid interface}

As outlined before, V2G devices are single-phase ones. The control approach adopted to manage its electronic interface is based on a PQ control approach, where the reactive power injection is set to zero. The active power set point (producing or charging) defined for this control represents the power exchange between the batteries and the grid. The single-phase inverter is modelled and controlled as presented in Ref. [10]. The active power set point is adapted to the grid operation conditions through a specific control, as presented in Section 5.

\subsection{Balancing unit}

Since LV microgrids will have to operate under three-phase unbalanced conditions (due to the connection of single-phase loads and/or microgeneration units), a three-phase VSI with a voltage balancing control mechanism was adopted in this research to improve system operation regarding system voltage unbalancing. The VSI unit interfaces with a storage device (such as battery or flywheel) and the MG, and has the ability to produce three independent output voltages, regardless of loading. The operating principle of the balancing unit is based on cancelling all unwanted negative and zero-sequence voltage-current components, according to the control structure described in Refs. [11,12]. In order to allow the operation under interconnected or islanded operation mode, it is necessary to provide a variable-frequency operation mechanism, which can be provided through the use of droop control concepts (active power versus frequency droop and reactive power versus voltage droop), as described in Ref. [5]. The use of droop control concepts allows the definition of the voltage reference for the voltage balancing unit [12].

\subsection{Other components}

The dynamic models adopted for microturbines and PV panels are the same as presented in previous work $[3,5,12]$. These components were considered to be grid interface through inverters with a PQ control approach.

The LV network is modelled as four-wire RL model. The load modelling adopted consists in a series of connected RL branches with constant impedances (as provided by the SimPower Library of Matlab Simulink).

\section{Control}

\subsection{Strategies for the $M G$}

Different control strategies for the operation of a MG have been studied and can be found in the literature [3,5]. For the islanded operation mode, two control schemes can be identified: the SMO and the MMO approach. In the SMO (Single Master Operation) a VSI - acting as master - is used as voltage reference when the MV network is lost; all other inverters are operated in PQ mode. The MMO (Multi-Master Operation) approach uses several inverters in a VSI mode with pre-defined frequency/active power voltage/reactive power characteristics. In these operating modes the MGCC can modify the generation profile by changing the idle frequency of the VSI(s) and/or define new set points for controllable microsources connected to the grid through PQ-controlled inverters [5].

In this study, the SMO approach was used, where the storage device presented in Fig. 2 acts as the "master-VSI" and all other sources are operated in a PQ like mode.

\subsection{V2G interfaces}

\subsubsection{Local control}

Several possibilities of local control to be housed in EV grid interfaces have been investigated. The observation variables used for this purpose correspond to frequency and voltages measured at 


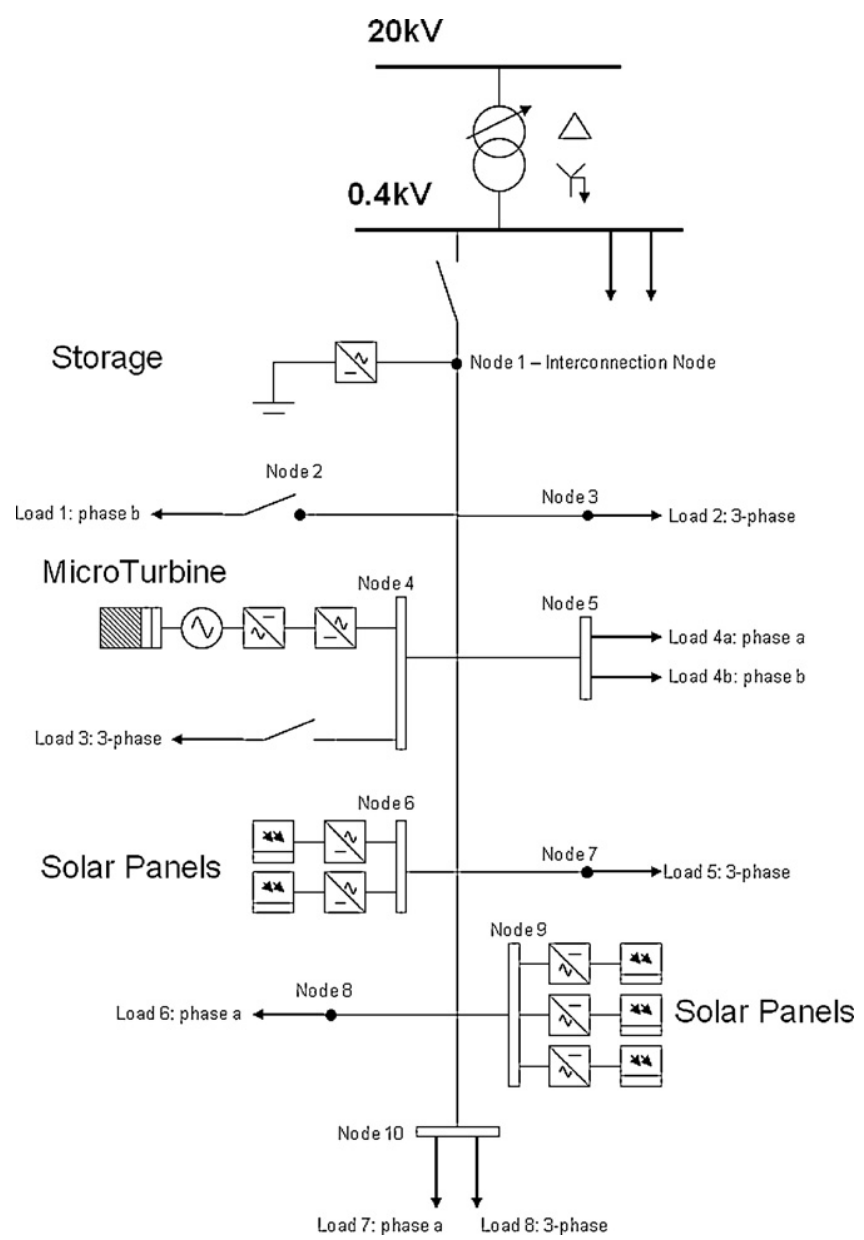

Fig. 2. Single line diagram of the investigated MG.

the EV connection terminal. In interconnected mode no frequency deviations from the nominal value $(50 \mathrm{~Hz})$ are considered.

In islanded mode, the frequency is an instantaneous indication for the power balance in the MG. It is therefore used to adapt the active power charging of the EV batteries. For this purpose the following control approach was developed:

- EV charge up their batteries, at nominal frequency (and above), by absorbing power at nominal charging rate $\left(P_{\mathrm{r}}\right.$ at $f_{\text {nom }}$ on Fig. 3(a)).

- The power consumption is linearly reduced to zero when the frequency drops below the nominal value. The zero-crossing frequency, $f_{0}$, is a parameter of free choice.

- If the system frequency drops below $f_{0}$, the devices start to inject power into the grid (becoming a V2G) device. At $f_{\text {neg, another }}$ parameter of free choice, the V2G device injects the maximum available power (Fig. 3(a)).

In this research the slopes of the power reduction above and below the zero-crossing frequency are set to be equal, as described by the dashed line in Fig. 3(a). For simplification purposes, the saturation is also made symmetrically (at $\pm P_{\mathrm{r}}$ ). This proportional control is inspired by the existing droop characteristics of the balancing unit and is therefore called "frequency droop control" of the V2G device. Nevertheless, it is important to notice that this control mechanism is implemented in an opposite way to the droop control implemented in the VSI/balancing unit, where power measurements are used for a frequency like control. In this case, a local measurement of grid frequency is taken by the V2G and is used in order to define a set point for the power exchange with the LV grid.

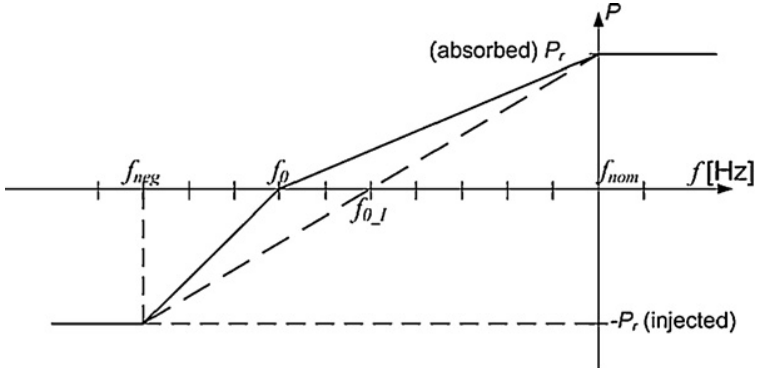

(a)

(b)

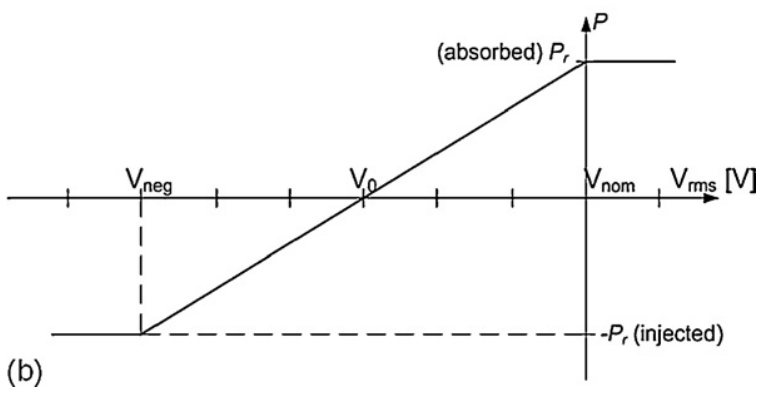

Fig. 3. Control settings of the V2G devices. (a) Frequency regulation and (b) voltage regulation.

The operating set point of the V2G device is then calculated from Eq. (6). In this equation $f_{0}$ corresponds to $f_{0 \_I}$ on Fig. 3(a).

$P_{\text {set_f }}=\frac{1}{k_{\mathrm{P}}} \cdot\left(f_{\text {Grid }}-f_{0}\right)$

where $f_{\text {Grid }}$ is the measured grid frequency, $f_{0}$ the zero-crossing frequency, and $k_{\mathrm{P}}$ defines the slope.

The selection of the most adequate values for $f_{0}$ (and $f_{\text {neg }}$ ) depends on the effectiveness of existing local secondary frequency control as well as on the local load shedding schemes. These parameters may differ from grid to grid and can be changed by the MGCC, using the communication link that is supposed to exist. This will require a communication protocol between the MGCC and V2G interfaces that needs to be further researched.

The MG is a LV network with lines having high $R / X$ coefficients. If voltage sags occur, it has been shown that they cannot be corrected efficiently by injecting reactive power. In the present case it is more efficient to reduce the load or inject active power. Therefore a similar control approach as the one used for frequency regulation can be implemented to deal with voltage drops that may result from normal or abnormal operation of the MG. Fig. 3(b) shows the used settings, and Eq. (7) gives the corresponding control law. Again two different slopes can be imagined for values above and below the zero-crossing voltage $V_{0}$.

$P_{\text {set_V }}=\frac{1}{K_{\mathrm{V}}} \cdot\left(V_{\text {phase }}-V_{0}\right)$

where $V_{\text {phase }}$ is the measured neutral-phase voltage, $V_{0}$ the zerocrossing voltage and $k_{\mathrm{V}}$ defines the slope. Again this control scheme involves saturation, as shown in Fig. 3(b).

A combination of these two control strategies can be done by defining the power set point to be followed by each V2G device. The adoption of a mean value approach is not optimal, as both parameters (frequency and voltage) would have to be simultaneously at the lower defined limit $\left(f_{\text {neg }}\right.$ and $V_{\text {neg }}$ in Fig. 3$)$ for the V2G device to inject its maximum available power. In islanding mode, load balancing should be considered as the key concern. Therefore the power set point to be adopted by each V2G device is defined in (8). In this way, local generation scarcity is taken into account with 

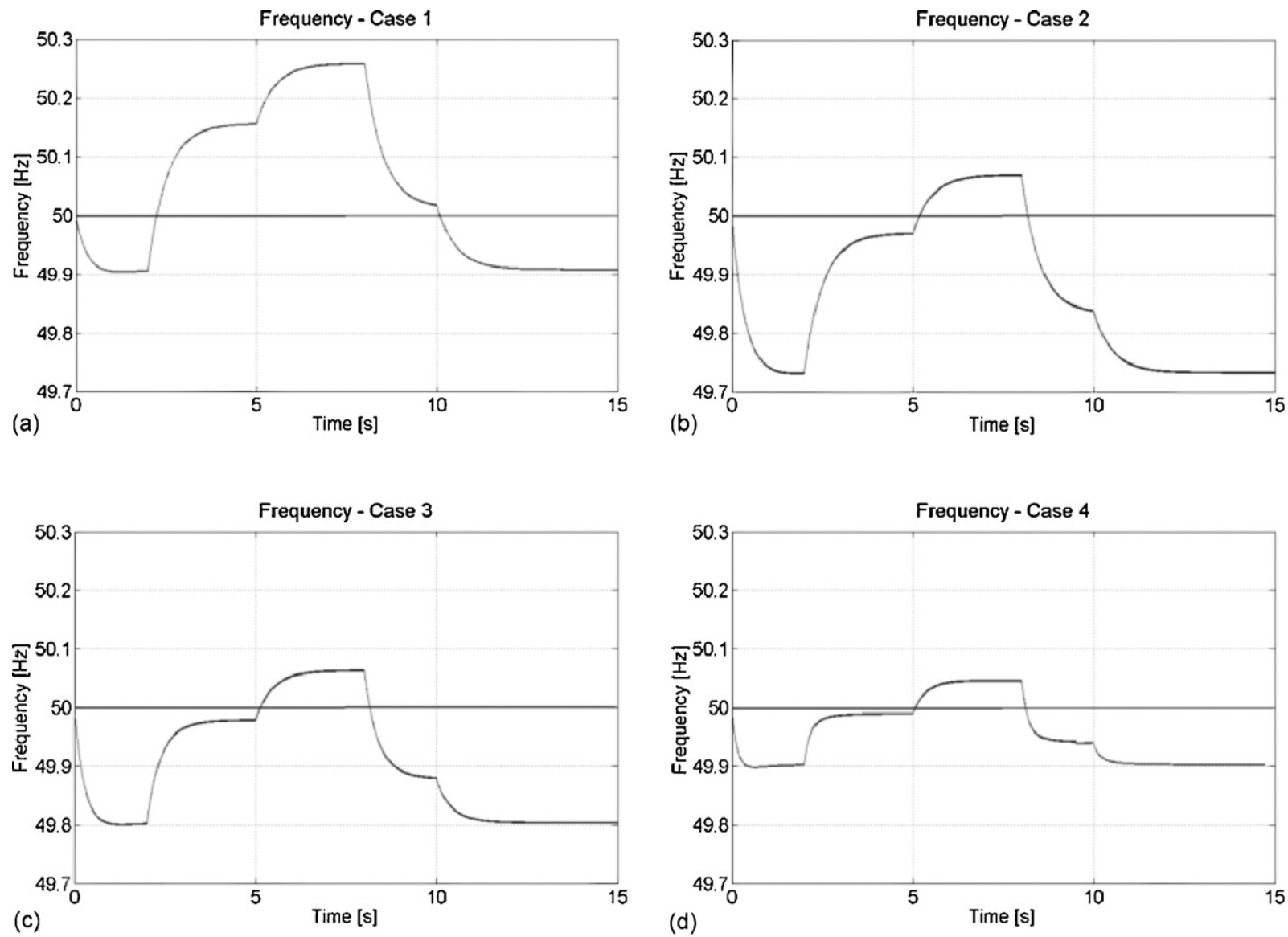

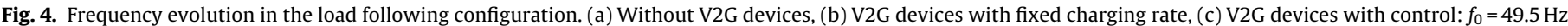
and (d) V2G devices with control: $f_{0}=49.9 \mathrm{~Hz}$.

priority regarding local voltage drops.

$P_{\text {set }}= \begin{cases}P_{\text {set } \_f} & \text { if } P_{\text {set_f }}<P_{\text {set_ }} \mathrm{V} \\ P_{\text {set_V }} & \text { otherwise }\end{cases}$

\subsubsection{Centralized control}

Another possibility to control the V2G interfaces is to send power set points from a central control device to each electric vehicle or group of EV. This control approach is used to balance single-phase loads in the MG by the means of the V2G interfaces. To implement this control, active power exchange between a threephase storage unit (e.g. the master unit) and the MG is measured in each phase. The set points sent to the EV connected in phases a-c in different nodes of the MG, are centrally calculated by the MGCC that acts in secondary control manner. The calculation of these power set points is shown in (9) and (10).

$e_{1}(t)=P_{\text {mes_a }}(t)-P_{\text {mes_b }}(t)$

$e_{2}(t)=P_{\text {mes_b }}(t)-P_{\text {mes_c }}(t)$

$e_{1}(t)=P_{\text {mes_c }}(t)-P_{\text {mes_a }}(t)$

$P_{\text {set_a }}(t)=\int k \cdot\left(e_{3}(t)-e_{1}(t)\right) \mathrm{d} t$

$P_{\text {set_b }}(t)=\int k \cdot\left(e_{1}(t)-e_{2}(t)\right) \mathrm{d} t$

$P_{\text {set_c }}(t)=\int k \cdot\left(e_{2}(t)-e_{3}(t)\right) \mathrm{d} t$ where $P_{\text {mes_x }}$ is the active power exchange value measured in phase $\mathrm{x}(\mathrm{a}-\mathrm{c})$, and $k$ is a coefficient used to speed up the time integration procedure.

The development of the expressions given in (10) in positive, negative and zero-sequence components shows that the set points $P_{\text {set_a }}, P_{\text {set_b }}, P_{\text {set_c }}$ reach a stable value only when all negative and zero-sequence voltages (and currents) become zero.

Eq. (10) is then saturated at \pm 1 . The set points sent to each V2G device in each phase are obtained from a proportional share of $P_{\text {set_a }}$ $P_{\text {set_b }}, P_{\text {set_c }}$ according to the rated power of each V2G device under control. This means that the MG communication infrastructure should be extended to the EV grid interface devices and each time an EV is plugged into the grid, information about its rated power and state of charge of its battery needs to be sent to the MGCC. It is assumed that only batteries with a pre-defined minimum state of charge would participate in this control.

\section{Test and simulation platform}

In order to test these control approaches the simulation platform described in Refs. [3,5] was adapted to incorporate the central balancing unit and the EV grid interface devices described before. Fig. 2 shows the single line diagram of the MG used in this research. A highly loaded MG, with single-phase and three-phase generation and loads, was exploited for testing purposes. V2G devices were assumed to be connected in parallel to each single-phase load.

This MG was shaped to be load unbalanced, with the majority of the single-phase load connected to phase a. In islanded mode, the frequency and voltage references are provided by the stor- 
Table 1

Connection scheme for the interruptible loads.

\begin{tabular}{lll}
\hline Simulation time [s] & Load 1 (single phase) & Load 2 (three phase) \\
\hline $0-2$ & $\mathrm{X}$ & $\mathrm{X}$ \\
$2-5$ & $\mathrm{X}$ & \\
$5-8$ & & $\mathrm{X}$ \\
$8-10$ & $\mathrm{X}$ & $\mathrm{X}$ \\
$10-15$ & $\mathrm{X}$ &
\end{tabular}

$\mathrm{X}$ denotes loads connected to the MG, blank means loads disconnected

Table 2

Charging levels of the V2G devices over the $15 \mathrm{~s}$ simulation in the load following configuration.

\begin{tabular}{lc}
\hline & Charging level \\
\hline Case (b) & $100 \%$ \\
Case (c) & $78.5 \%$ \\
Case (d) & $46.8 \%$ \\
\hline
\end{tabular}

age unit connected at the interconnection node. A SMO approach was adopted for control purposes. This storage unit has the ability to act as balancing unit and to provide three independent output voltages.

Different control type approaches were used to test the impact of the V2G devices in the MG. These control approaches and the obtained results are described in the next sections.
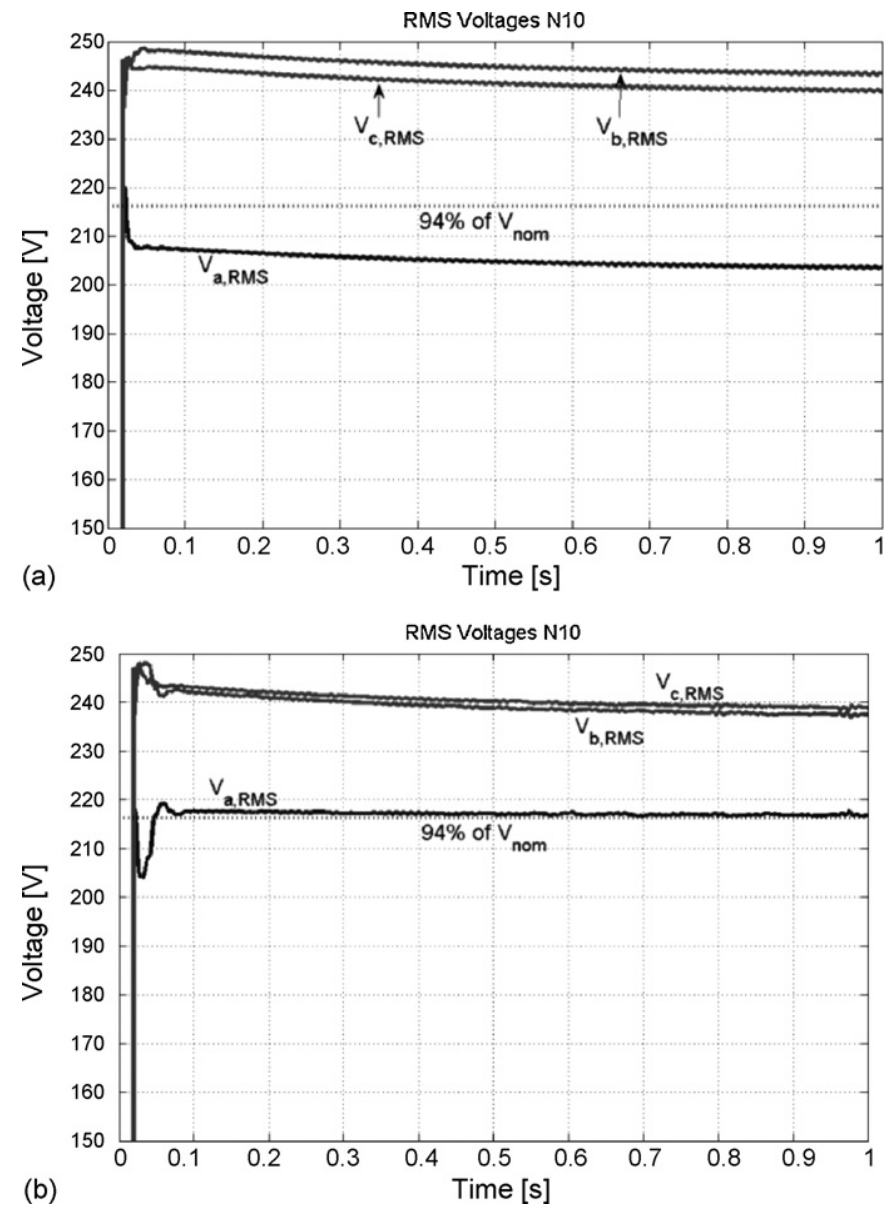

Fig. 5. Voltages at node 10 when including frequency and voltage droop controlled V2G devices in islanded mode. (a) No V2G devices, profile 1 and (b) including V2G devices, profile 1 .
Table 3

Energy stored in the V2G devices during MG islanded operation.

\begin{tabular}{lc}
\hline & Profile 1 [pu] \\
\hline Node 2 & 0.816 \\
Node 5, phase a & 0.407 \\
Node 5, phase b & 0.556 \\
Node 8 & -0.814 \\
Node 10 & -0.814 \\
\hline
\end{tabular}

\section{Results and discussion}

\subsection{Islanded operation mode}

At least one master-storage unit has to be present to allow the islanded MG operation. The balancing unit was assumed to be in operation in all the simulations performed. This unit ensures the load-generation-balance by injecting or absorbing the power difference and is capable of balancing the voltages at the interconnection node. Load profile 1 (Table 4 in Appendix A) is used for simulation purposes.

\subsubsection{Frequency droop mode - load following}

In this section, the V2G devices are operated in frequency droop control mode only, as the first investigated issue concerns the MG frequency behaviour. To identify the influence of these devices, four different cases were studied:
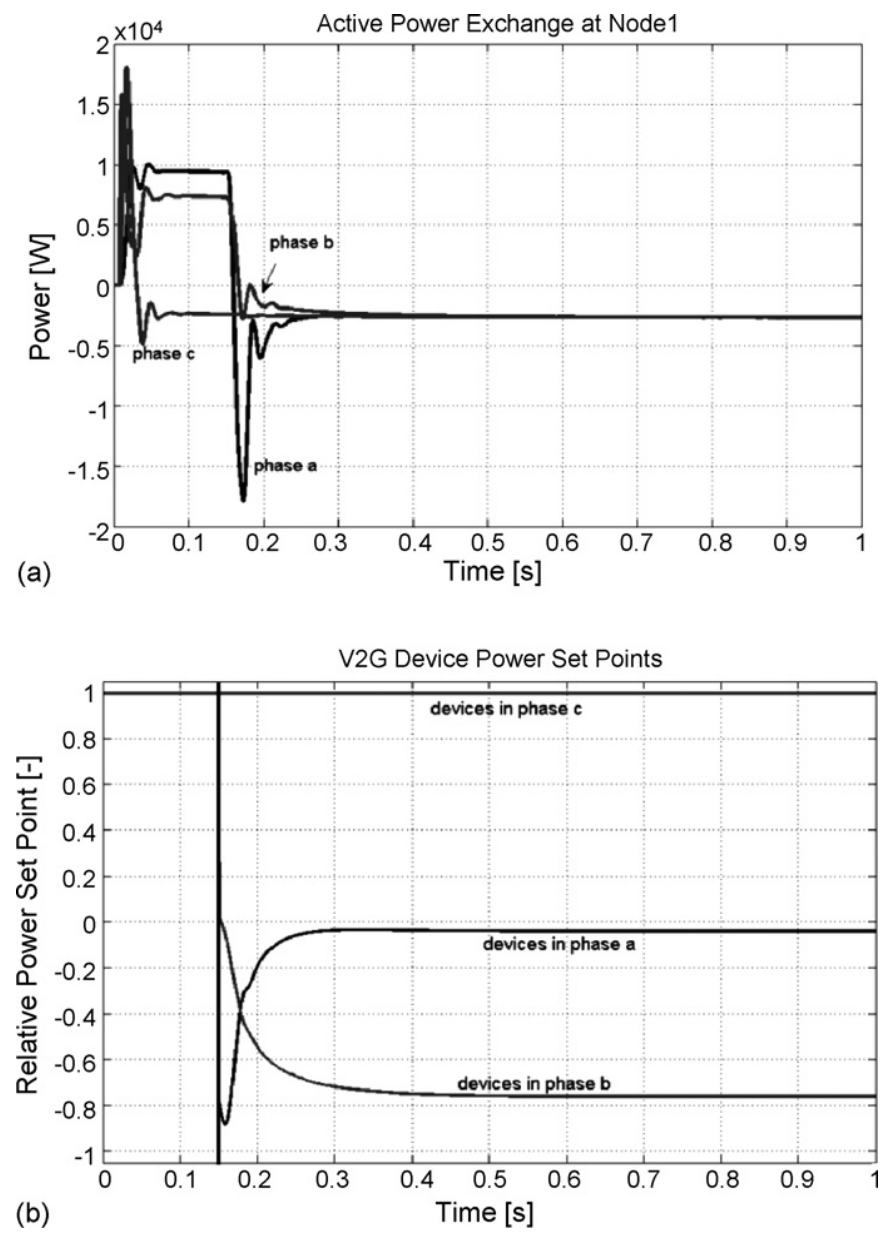

Fig. 6. Centralized V2G control, including balancing unit. (a) Power exchange per phase, node 1 and (b) power set point for the V2G devices. 
(a) MG without V2G devices.

(b) V2G devices connected with a fixed charging rate.

(c) V2G devices controlled with $f_{0}=49.5 \mathrm{~Hz}$.

(d) V2G devices controlled with $f_{0}=49.9 \mathrm{~Hz}$.

The effect on the grid frequency is shown in Fig. 4. One single-phase and one three-phase load were disconnected and reconnected, during the simulation, as described in Table 1 . The initial frequency drop is due to islanding from the MV network (local load is larger than generation): it was assumed that for all $t \leq 0 \mathrm{~s}$ the MG is in the interconnected mode, and therefore the frequency is $50 \mathrm{~Hz}$.

In case (a), it can be observed that during most of the time, the generation exceeds the load. It is therefore possible to add, at least in the low load period, some EV charging. If V2G devices with a fixed charging rate are included, the frequency shifts to a lower level, as can be seen in Fig. 4(b). The main storage unit is injecting power for frequency values below $50 \mathrm{~Hz}$ and absorbing power for values above $50 \mathrm{~Hz}$. In case (b), this unit has therefore to inject energy to the grid. Adapting the load dynamically when the frequency falls below $50 \mathrm{~Hz}$ is therefore most appropriate, as it reduces the totally required energy by the storage unit. In Fig. 4(c) and (d) it can be observed that the system frequency can be indirectly influenced by choosing the value of the zero-crossing frequency, $f_{0}$. This control also influences the amount of energy stored in each device, as can it be observed in Table 2. For the values in Table 2 it was assumed, that charging at nominal current during the whole simulation time equals $100 \%$. Nevertheless the maximal charging rate may not be required in all situations. To decide what amount of charging is

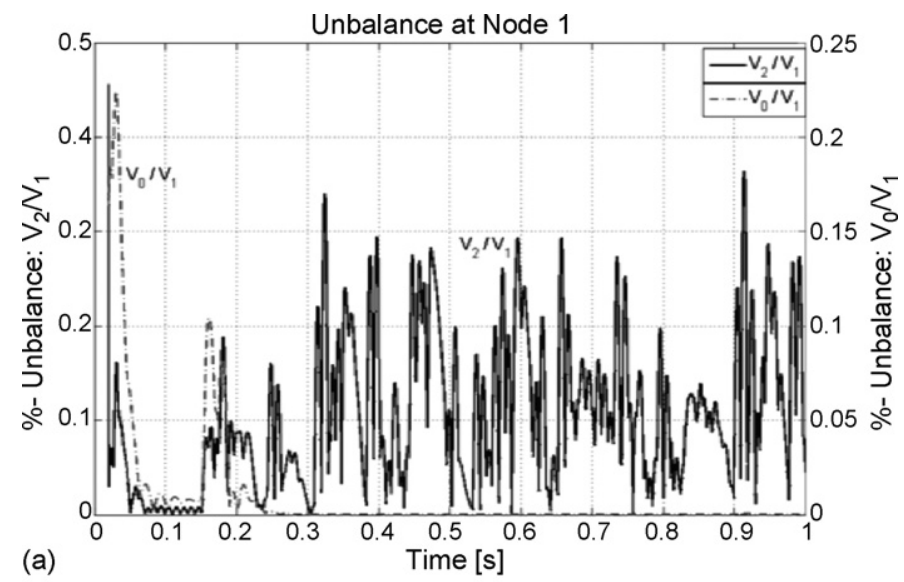

(a)
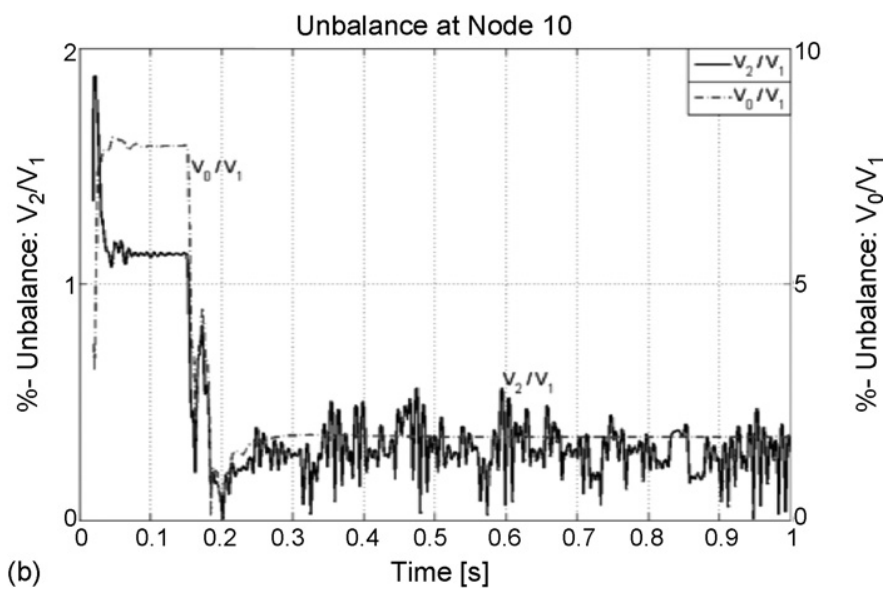

Fig. 7. Unbalance levels with centralized V2G control and balancing unit. (a) Node 1 and (b) node 10 . necessary, some factors may have to be considered, like remaining energy in the battery, time of the day, scheduled travels, etc.

\subsubsection{Frequency and voltage droop mode}

In this case only the V2G device control mode was changed. The other settings of the MG were maintained. This means that the balancing unit ensures load-generation-balance and has the capability for voltage balancing at the interconnection node. The V2G device control includes now also the described voltage droop control approach. The power set points of the frequency and voltage droop control are combined as presented in (8).

It was observed that the voltage level in phase a in the lower part of the investigated MG sinks below $94 \%$ of the nominal value (see Fig. 5(a)). This threshold was defined as being the value where the V2G devices inject their maximum power when controlled in a voltage droop mode ( $V_{\text {neg }}$ in Fig. 3(b)). The presence of V2G devices in this network phase contributes to improve the voltage level. This is shown in Fig. 5(b). It can be observed that the presence of the V2G devices raises considerably the voltage level. It can be seen that the V2G devices in this control configuration are capable of fast reacting to voltage sags.

Table 3 indicates the fraction of energy stored in the EV batteries over the whole simulation time, where " 1 " represents charging the battery during all the time at nominal charging rate; negative values stand for injecting energy into the grid. In this table it can be observed that some devices inject more than $80 \%$ of their rated capacity. On the other hand, at locations in the grid where the voltage level is not a critical issue the connected V2G devices are still capable of charging.
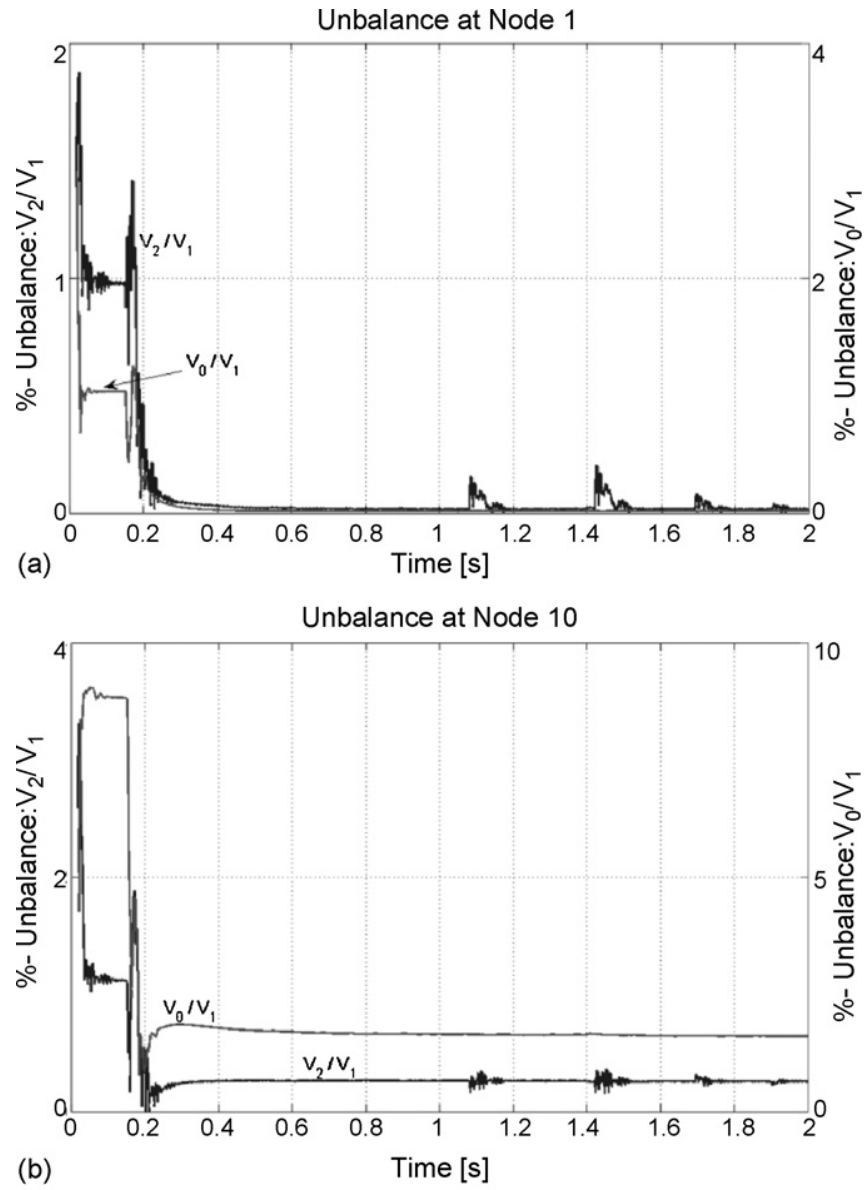

Fig. 8. Unbalance levels with centralized V2G control, including a storage unit without balancing mechanism. (a) Node 1 and (b) node 10 . 


\subsection{Centralized control}

In the following set of tests the load profile was slightly modified. The load in the lower part of the MG, nodes 8 and 10, was reduced in order to increase the voltage level (see Table 4 in Appendix A for details of profile 2 ). The control of the V2G devices is done only in a centralized way, as presented in Section 5. In order to increase the simulation speed, the (local) frequency and voltage droop control mode of the V2G devices was not maintained. Simulations showed that the proposed control can only work efficiently if it is possible to influence the load in all three phases; hence a V2G device in phase $c$ has been added. The control starts to act after $t=0.15 \mathrm{~s}$, when the transients due to initial conditions are settled.

\subsubsection{Islanded balanced operation}

As previously mentioned, for these set of tests a second load profile - profile 2 - was used. The balancing unit was also connected to the MG, ensuring the load-generation-balance and balancing voltages at the interconnection node.

In Fig. 6(b) can be observed, that the V2G devices in phase c are asked to charge at their maximum rate, as the single-phase load in phase $\mathrm{c}$ is lower than in the other phases. The power exchange rates in each phase between the balancing unit and the grid are therefore set equal to the one in phase c (see Fig. 6(a)).

It is interesting to observe the unbalance levels in the grid. At the interconnection node (node 1 ), this level is not affected positively. As can be seen in Fig. 7(a), the zero-sequence voltage is reduced to
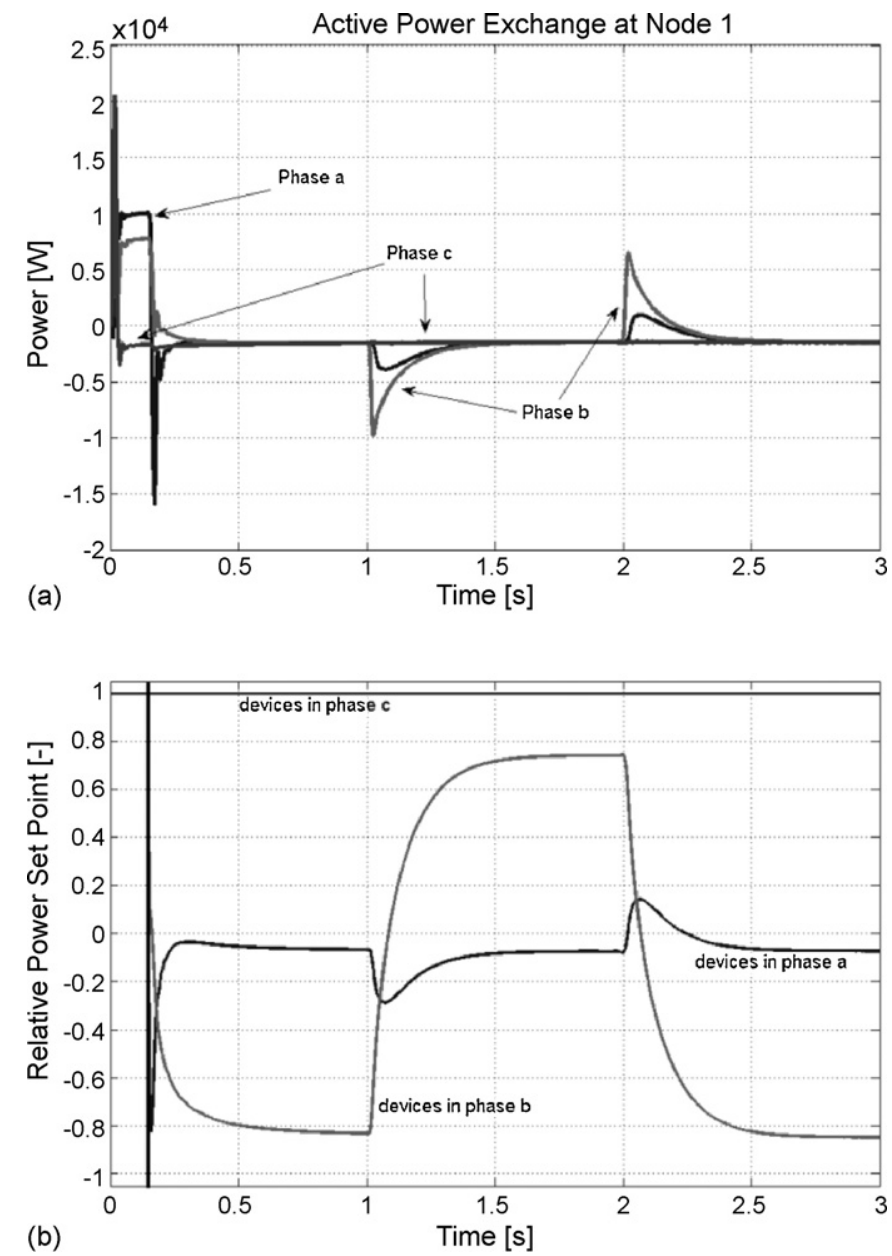

Fig. 9. Centralized V2G operation in the load following case. (a) Power exchange per phase, Node 1 and (b) power set point for the V2G devices. zero (all single-phase load is balanced), but the negative sequence voltage is increased. On the other hand, in the lower part of the grid (e.g. at node 10, Fig. 7(b)), the unbalance is considerably reduced.

\subsubsection{Islanded operation including a storage device without balancing mechanism}

As it was seen before, the centralized control and the balancing unit are not working well together, even though their objective is the same: balance the voltage in the grid. This is due to the different control approaches used. It is therefore interesting to investigate the efficiency of the centralized control, when only a simple storage device is connected to the MG, without voltage balancing mechanism. Therefore in the set of tests that are described next the voltage balancing mechanism described in Section 4 is not in operation. Nevertheless the simple storage unit used for load-generation balance purposes has the same rated power as the balancing unit in the simulations described before.

The results with respect to power exchange at node 1 and the power set points are very similar to the plots shown in Fig. 6 and were therefore omitted. On the other hand, the unbalance level presents some interesting differences: it can be observed in Fig. 8(a) that the unbalance at node 1 is reduced to (almost) zero. This shows that this control has also the same effect as the previously used balancing unit. The main advantage of this control compared with the use of a balancing unit is shown in Fig. 8(b): it affects the whole MG. The unbalance is also considerably reduced in the lower part of the MG and not only in the part electrically close to the interconnection node, where the balancing unit was located.

7.2.2.1. Load following. The storage device connected to the MG ensures the load following. All loads can always be served and a
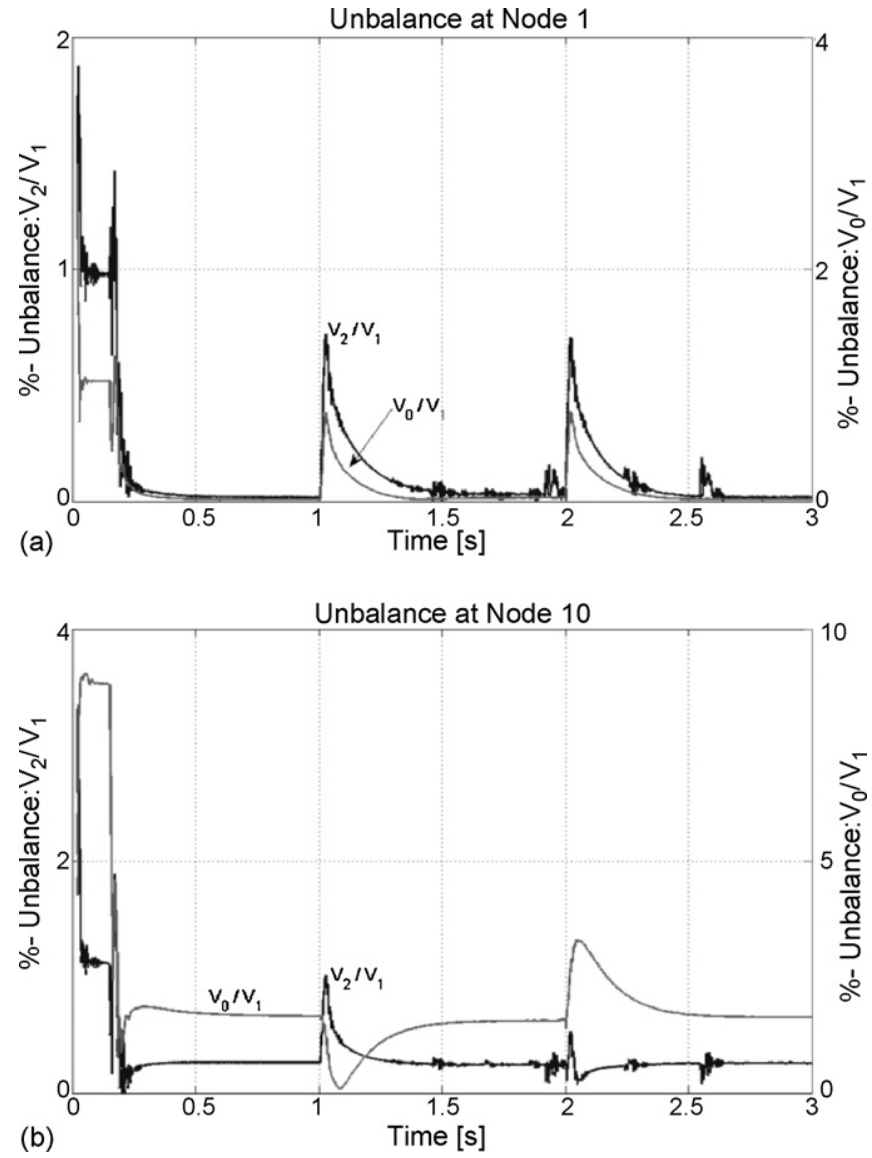

Fig. 10. Unbalance levels in the load following case. (a) Node 1 and (b) node 10. 
Table 4

Load and generation profiles.

\begin{tabular}{lll}
\hline & Profile $1[\mathrm{~kW} / \mathrm{kVar}]$ & Profile $2[\mathrm{~kW} / \mathrm{kVar}]$ \\
\hline Load 1 & $8 / 0.5$ & $8 / 0.5$ \\
Load 2 & $10 / 5$ & $10 / 5$ \\
Load 3 & $20 / 5$ & $20 / 5$ \\
Load 4a & $5 / 0.1$ & $5 / 0.1$ \\
Load 4b & $3 / 0.1$ & $3 / 0.1$ \\
Load 5 & $20 / 1$ & $20 / 1$ \\
Load 6 & $10 / 0.5$ & $2 / 0.1$ \\
Load 7 & $8 / 2$ & $1.6 / 0.4$ \\
Load 8 & $10 / 5$ & $2 / 1$ \\
Storage device & $80 / 30$ & $80 / 30$ \\
Microturbine & $80 / 30$ & $80 / 30$ \\
SP: node 6, phase a & $1.5 / 0$ & $1.5 / 0$ \\
SP: node 6, phase b & $1.2 / 0$ & $1.2 / 0$ \\
SP: node 9, phase a & $1.5 / 0$ & $1.5 / 0$ \\
SP: node 9, phase b & $1 / 0$ & $1 / 0$ \\
SP: node 9, phase c & $0.8 / 0$ & $0.8 / 0$ \\
\hline
\end{tabular}

a Rated capacity; labels refer to Fig. 2; SP stands for solar PV panel.

frequency reference is provided. For simulating the load following behaviour of V2G devices, a single-phase load connected to phase $\mathrm{b}$ was disconnected and reconnected (at $t=1$ and $t=2 \mathrm{~s}$ ).

The power exchange per phase of the storage device is shown in Fig. 9(a) and the set points of the V2G devices are described in Fig. 9(b). It can be observed, that the set point for the devices in phase $b$ is modified in order to rebalance the new load configuration. The speed to achieve this can be influenced by choosing the coefficient $k$ in (10). In Fig. 10(a) and (b) the unbalance levels at nodes 1 and 10 are shown.

\section{Conclusion}

From the research developed it was possible to conclude that if EV grid interfaces include a local control, reacting to frequency and voltage deviations at the point of connection, it is easy to integrate EV into LV distribution grids and even get technical benefits for MG operation, namely for islanding operation modes.

Appropriate MG operation can be assured if each EV interface device is controlled in a so-called "frequency-voltage-droop" mode. The frequency during islanded operation can be positively influenced and be kept closer to its nominal value with the presence of V2G devices. By adapting the control parameters of the EV grid interfaces to current operation conditions, such as the zerocrossing frequency, better performance results can be obtained than with a static set of control settings. These settings may also differ from MG to MG. In islanding mode the central storage unit, used to balance the MG, can have a smaller dimension since the distributed storage resources housed in the EV batteries can be used to help manage the system, playing an important role in balancing the load in the MG. By controlling the EV grid interface devices centrally, the power quality throughout the whole grid (namely voltage unbalance levels) can also be improved.

\section{Acknowledgment}

J. A. Peças Lopes and C. L. Moreira want to express their gratitude to Fundação para a Ciência e Tecnologia (Portugal) for supporting the research work presented in this paper (Grant PTDC/EEAEEL/103546/2008).

\section{Appendix A.}

Table 4 lists the MG load profiles used for simulation purposes (both single phase and three phase), together with some microsources characteristics.

\section{References}

[1] W. Kempton, J. Tomić, Vehicle-to-grid power fundamentals: calculating capacity and net revenue, J. Power Sources 144 (2005) 268-279.

[2] A.N. Brooks, Vehicle-to-grid Demonstration Project: Grid Regulation Ancillary Service with a Battery Electric Vehicle, AC Propulsion Inc., 2002.

[3] J.A. Peças Lopes, C.L. Moreira, F.O. Resende, Microgrids black start and islanded operation, in: Proc. 15th PSCC, 2005

[4] N.J. Gil, J.A. Peças Lopes, Hierarchical frequency control scheme for islanded multi-microgrids operation, in: Proc. IEEE Lausanne Power Tech, 2007.

[5] J.A. Peças Lopes, C.L. Moreira, A.G. Madureira, Defining control strategies for microgrids islanded operation, IEEE Trans. Power Syst. 21 (2006) 916-924.

[6] F. Katiraei, R. Iravani, N. Hatziargyriou, A. Dimes, Microgrids management, IEEE Power Energy Mag. 6 (2008) 54-65.

[7] K. Parks, P. Denholm, T. Markel, Costs and emissions associated with plug-in hybrid electric vehicle charging in the Xcel Energy Colorado Service Territory, Technical Report NREL/TP-640-41410, May 2007.

[8] A. von Jouanne, B. Banerjee, Assessment of voltage unbalance, IEEE Trans. Power Deliv. 16 (2001) 782-790.

[9] R.H. Lasseter, P. Piagi, Control and design of microgrid components, PSERC Technical Report, Publication 06-03, January 2003.

[10] B. Burger, A. Engler, Fast signal conditioning in single phase systems. Available online: http://www.iset.uni-kassel.de/abt/FB-A/publication/2001/ 2001graz_engler_epe.pdf.

[11] Y. Li, D.M. Vilathgamuwa, P.C. Loh, Microgrid power quality enhancement using a three-phase four-wire grid-interfacing compensator, IEEE Trans. Ind. Appl. 41 (2005) 1707-1719.

[12] U. Zahnd, Control strategies for load-following unbalanced microgrids islanded operation, Master's Thesis, EPF Lausanne/INESC Porto, February 2007. Available online: http://vpaa.epfl.ch/webdav/site/vpaa/shared/ Prix\%20de\%20recherche\%2007/Master\%20Thesis\%20U.\%20Zahnd.pdf.

J.A. Pecas Lopes received the electrical engineering degree and the Ph.D. degree in electrical engineering from the University of Porto, Porto, Portugal, in 1981 and 1988 , respectively. In 1996, he received the aggregation degree from the University of Porto. Currently, he is full professor in the Department of Electrical Engineering of the Faculty of Engineering of University of Porto and a member of the Board of Directors of INESC Porto - Instituto de Engenharia de Sistemas e Computadores do Porto.

Silvan A. Polenz obtained the M.Sc. degree in electrical and electronic engineering from the Swiss Federal Institute of Technology in Lausanne (EPFL), Switzerland. His research activities for the M.Sc. degree were developed at the Power System Unit of INESC Porto, where he had the opportunity to integrate a research group on microgeneration, microgrids and electric vehicles integration in distribution grids. Currently he is with Stadler Bussnang AG, Switzerland.

C.L. Moreira is a senior researcher in the Power Systems Unit of Instituto de Engenharia de Sistemas e Computadores do Porto (INESC Porto). He obtained the electrical engineering degree (5-year course) in 2003 and the $\mathrm{Ph}$. D in 2008, both from the Faculty of Engineering of Porto University, Porto, Portugal. His main research interests are focused on microgrids dynamics and control, smart grids and smart metering.

Rachid Cherkaoui received both the M.Sc. and Ph.D. degrees in electrical engineering in 1983 and 1992, respectively, from the Swiss Federal Institute of Technology in Lausanne (EPFL), Switzerland. He is now a senior research engineer at EPFL, LRE. His research interests are in electricity market deregulation, distributed generation and storage, and power system vulnerability mitigation. He is a member of technical program committees of various conferences, member of CIGRE TFs and WGs, and IEEE chapter officer since 2005 . He is the author and co-author of more than 50 scientific publications. 